\title{
Study of The Podocalyxin as An Early Marker for Diabetic Nephropathy and Its Correlation with Stages of Diabetic Nephropathy in A Sample of Egyptian Patients with T2DM
}

Salah El-Din Ahmed Shelbaya, Rana Hashem Ibrahim, Nourhan Safwat Sawirs, Hanan Mahmoud Ali* Department of Internal Medicine and Endocrinology, Faculty of Medicine, Ain Shams University, Cairo, Egypt *Corresponding author: Hanan Mahmoud Ali, Mobile: (+20)1222521314, Email: hanan.mahmoud29@yahoo.com

\begin{abstract}
Background: Nephropathy is a major complication of diabetes, and is the main cause of end-stage kidney disease. Early recognition of nephropathy in diabetic patients is critical because it creates an opportunity to prevent the occurrence of diabetic nephropathy and/or even slow down the process of end-stage kidney disease attributable to diabetes. Human cells; podocytes (pods) have been shown to be functionally and structurally affected in the natural history of diabetic nephropathy.

Objective: Study of the podocalyxin as an early marker for diabetic nephropathy and its correlation with stages of diabetic nephropathy in a sample of Egyptian patients with type 2 diabetes mellitus (T2DM).

Patients and Methods:This study was a cross sectional study conducted on 80 subjects divided into four groups: Group 1: 20 diabetic patients with normalbuminuria. Group 2: 20 diabetic patients with nephropathy and microalbuminuria stages. Group 3: 20 diabetic patients with nephropathy and macroalbuminuria stages, Group 4:20 apparently healthy volunteers were included as a control group.

Results: Urinary podocalyxin (PCX) was significantly higher in patients group compared to control group. Urinary PCX was significantly higher in macroalbuminuric group followed by microalbuminuric group then normoalbuminuric group. There was a positive significant correlation between urinary PCX and glycemic profile [Fasting blood sugar (FBS), 2hrPP, Glycosylated Hemoglobin (HbA1C)], serum creatinine and albumin creatinine ratio (ACR).

Conclusion: Urinary podocalyxin is a beneficial as an early marker for early stages of diabetic nephropathy in type 2 DM patients.
\end{abstract}

Keywords: Diabetic nephropathy, Podocalyxin, T2DM.

\section{INTRODUCTION}

Diabetes is a group of metabolic diseases characterized by high blood sugar caused by defects in insulin secretion, insulin action, or both. Chronic hyperglycemia with diabetes is associated with longterm damage, dysfunction, and failure of various organs, especially the eyes, kidneys, nerves, and heart and blood vessels ${ }^{(1)}$.

Nephropathy is a major complication of diabetes and the main cause of end-stage kidney disease. Diabetic kidney disease in diabetes mellitus occurs in $20-40 \%$ of diabetic patients, and is clinically characterized by proteinuria and progressive renal insufficiency ${ }^{(2)}$.

Human cells (pods) have been shown to be functionally and structurally affected in the natural history of diabetic nephropathy. Podocytes cover the glomerular basement membrane (GBM) schema to contribute to the final volume and shipping barrier of the glomerular filter to completely reduce the leakage of proteins into the urinary space ${ }^{(3)}$.

Injuries of podocytes are associated with a hiatus, loss of incision membranes, erasure of the foot process, and detachment from GBM ${ }^{(4)}$.

Three noteworthy tissue changes occur in the glomeruli in people with diabetic nephropathy. Firstly, mesangial expansion induced by hyperglycemia, which is likely by increased production of matrix or sugar in matrix proteins. Second, a thickening of the glomerular basement membrane (GBM) occurs. Third, glomerular sclerosis is caused by high blood pressure within the glomeruli (caused by dilatation of the incoming renal artery or from ischemic damage caused by stenosis of hyaline in the vessels supplying the glomeruli) ${ }^{(5)}$.

Podocalyxin, the sialomucin most closely related to cluster of differentiation 34 (CD34) and endoglycan, is expressed by kidney podocytes, hematopoietic progenitors, and vascular endothelium. The function of PCX is to maintain the shape of a podocyte. PCX is usually found on the apical cell membrane of podocytes and is shed into urine from infected PADS cells ${ }^{\left({ }^{(6)}\right.}$.

\section{AIM OF THE WORK}

Study of the podocalyxin as an early marker for diabetic nephropathy and its correlation with stages of diabetic nephropathy in a sample of Egyptian patients with T2DM.

\section{PATIENTS AND METHOD}

This is a cross-sectional study that was performed during the period between April and December 2018; conducted on patients attending the diabetes outpatient clinics in Ain Shams University hospitals. 
Ethical approval and written informed consent:

An approval of the study was obtained from Ain Shams University academic and ethical committee.

Every patient signed an informed written consent for acceptance of the operation.

The study was conducted on 80 subjects divided into four groups:

Group 1: 20 patients with T2DM with norm albuminuria. Group 2: 20 patients with T2DM with nephropathy and microalbuminuria stages.

Group 3: 20 patients with T2DM with nephropathy and macroalbuminuria stages, Group 4:20 apparently healthy volunteers (matched age and sex) were included as a control group. Their age ranged between 30-50 years, male and female patients with T2DM diagnosed according to the American Diabetic Association (ADA) 2018 diagnosis of diabetes criteria, excluding any patients with any history of cardiac, renal or vascular disease.

All subjects underwent the following: full medical history including age and type of diabetes medications, physical examination including blood pressure measurement, laboratory investigations including; serum creatinine, FBS, 2hrPP, HbAlc, albumin, albumin/creatinine ratio, urinary podocalyxin by enzyme-linked immune sorbent assay (ELISA).

\section{Statistical analysis}

Statistical presentation and analysis of the present study was conducted, using the mean, standard deviation, one-way analysis of variance [ANOVA] test, correlation coefficient (r) and multivariate regression analysis by SPSS V20. P-value $>0.05$ Non significant; P-value $\leq 0.05$ Significant; P-value < 0.001 Highly Significant.

\section{RESULTS}

The current study showed that there is a highly statistical significant difference between the studied groups as regard serum creatinine, ACR, podocalyxin, and glycemic profile being highest in group 3 followed by group 2 , then group 1 , then group 4 as shown in table (1).

Table (1): Comparison between the studied groups as regard laboratory data using ANOVA test

\begin{tabular}{|c|c|c|c|c|c|c|c|}
\hline & & $\begin{array}{c}\text { Group 1 } \\
\text { (DM with } \\
\text { Norm- } \\
\text { albuminuria) } \\
\mathbf{N}=\mathbf{2 0}\end{array}$ & $\begin{array}{c}\text { Group 2 } \\
\text { (DM with } \\
\text { micro- } \\
\text { albuminuria) } \\
\mathrm{N}=\mathbf{2 0}\end{array}$ & $\begin{array}{c}\text { Group 3 } \\
\text { (DM with } \\
\text { macro- } \\
\text { albuminuria) } \\
\mathrm{N}=20\end{array}$ & $\begin{array}{l}\text { Group 4 } \\
\text { (Control) } \\
\mathbf{N}=20\end{array}$ & $\mathbf{F}$ & P-value \\
\hline \multirow{2}{*}{$\begin{array}{c}\text { S. Create } \\
(\mathrm{mg} / \mathrm{dl})\end{array}$} & Range & $0.7-1.3$ & $0.8-1.4$ & $1-1.6$ & $0.5-1$ & \multirow[b]{2}{*}{40.131} & \multirow[b]{2}{*}{$<0.001 *$} \\
\hline & Mean \pm SD & $1.025 \pm 0.165$ & $1.170 \pm 0.187$ & $\begin{array}{c}1.335 \pm \\
0.173 \\
\end{array}$ & $\begin{array}{c}0.740 \pm \\
0.188 \\
\end{array}$ & & \\
\hline \multirow[b]{2}{*}{ FBS (mg/dl) } & Range & $129-170$ & $156-233$ & $210-256$ & $78-110$ & \multirow[b]{2}{*}{400.659} & \multirow[b]{2}{*}{$<0.001 *$} \\
\hline & Mean \pm SD & $146.900 \pm 11.832$ & $\begin{array}{c}184.250 \pm \\
16.998\end{array}$ & $\begin{array}{c}235.70 \pm \\
13.083\end{array}$ & $\begin{array}{c}94.400 \pm \\
10.525\end{array}$ & & \\
\hline \multirow[b]{2}{*}{$\begin{array}{l}2 \mathrm{Hr} \text { PP } \\
(\mathrm{mg} / \mathrm{dl})\end{array}$} & Range & $180-240$ & 240-299 & 263-320 & 110-149 & \multirow[b]{2}{*}{566.915} & \multirow[b]{2}{*}{$<0.001 *$} \\
\hline & Mean \pm SD & $197.550 \pm 13.450$ & $\begin{array}{c}254.600 \pm \\
12.296\end{array}$ & $\begin{array}{c}280.900 \pm \\
14.197\end{array}$ & $\begin{array}{c}129.900 \pm \\
9.963\end{array}$ & & \\
\hline \multirow[b]{2}{*}{ HbA1c \% } & Range & $6.5-7.1$ & $7.7-8.2$ & $8.7-10.5$ & $4.7-5.4$ & \multirow[b]{2}{*}{710.009} & \multirow[b]{2}{*}{$<0.001 *$} \\
\hline & Mean \pm SD & $6.850 \pm 0.179$ & $\begin{array}{c}7.900 \pm \\
0.162\end{array}$ & $\begin{array}{c}9.525 \pm \\
0.555\end{array}$ & $\begin{array}{c}5.035 \pm \\
0.181\end{array}$ & & \\
\hline \multirow[b]{2}{*}{$\operatorname{ACR}(\mathrm{mg} / \mathrm{g})$} & Range & $10.1-21.1$ & $37-171.7$ & $342-766$ & $10-22$ & \multirow[b]{2}{*}{208.743} & \multirow[b]{2}{*}{$<0.001 *$} \\
\hline & Mean \pm SD & $17.650 \pm 2.919$ & $\begin{array}{c}85.390 \pm \\
7.286\end{array}$ & $\begin{array}{c}462.250 \pm \\
23.562\end{array}$ & $\begin{array}{c}15.530 \pm \\
3.565\end{array}$ & & \\
\hline \begin{tabular}{|c|} 
Podocalyxin \\
(ngldl)
\end{tabular} & Mean \pm SD & $1.025 \pm 0.299$ & $\begin{array}{l}1.970 \pm \\
0.535 \\
\end{array}$ & $6.005 \pm 0.759$ & $\begin{array}{c}0.065 \pm \\
0.009 \\
\end{array}$ & 570.793 & $<0.001 *$ \\
\hline
\end{tabular}

On correlating urinary podocalyxin with all variables done in the current study, we found that there is a statistical highly significant positive correlation between urinary podocalyxin and serum creatinine, FBS, $2 \mathrm{HrPP}$, $\mathrm{HbA1c}$ and ACR and there is a statistical significant positive correlation between podocalyxin with age as shown in table (2). 
Table (2): Correlation between urinary PCX and other laboratory finding in all 80 subjects:

\begin{tabular}{|c|c|c|}
\hline \multirow{2}{*}{} & \multicolumn{2}{|c|}{ Correlations } \\
\cline { 2 - 3 } & PODOCALYXIN (ng/ml) \\
\hline Age (years) & 0.374 & P-value \\
\hline SBP (mmHg) & 0.116 & $\mathbf{0 . 0 0 3}$ \\
\hline DBP (mmHg) & 0.211 & 0.377 \\
\hline BMI (kg/m $\mathbf{2})$ & 0.421 & 0.106 \\
\hline S. Create (mg/dl) & 0.536 & 0.225 \\
\hline FBS (mg/dl) & 0.881 & $<\mathbf{0 . 0 0 1 *}$ \\
\hline 2Hr PP (mg/dl) & 0.782 & $<\mathbf{0 . 0 0 1 *}$ \\
\hline HbA1c \% & 0.895 & $<\mathbf{0 . 0 0 1} *$ \\
\hline ACR (mg/g) & 0.928 & $<\mathbf{0 . 0 0 1 *}$ \\
\hline
\end{tabular}

By applying multivariate linear regression to the statistically significant factors, we found that ACR is the most significant factor in comparison to podocalyxin, FBS is significant factor in comparison to podocalyxin and $\mathrm{HbA1c}$ is significant factor in comparison to podocalyxin as shown in table (3).

Table (3): Showing multivariate regression analysis between urinary PCX and other laboratory finding:

\begin{tabular}{|c|c|c|c|c|c|}
\hline & \multicolumn{2}{|c|}{$\begin{array}{c}\text { Unstandardized } \\
\text { Coefficients }\end{array}$} & \multirow{2}{*}{$\begin{array}{c}\begin{array}{c}\text { Standardized } \\
\text { Coefficients }\end{array} \\
\text { Beta } \\
\end{array}$} & \multirow[t]{2}{*}{$\mathbf{t}$} & \multirow[t]{2}{*}{ P-value } \\
\hline & $\mathbf{B}$ & Std. Error & & & \\
\hline Age & 0.001 & 0.021 & 0.001 & -0.002 & 0.999 \\
\hline S. Create (mg/dl) & 0.556 & 0.550 & 0.053 & 1.011 & 0.317 \\
\hline FBS (mg/dl) & 0.021 & 0.006 & 0.363 & 3.201 & $0.002 *$ \\
\hline 2Hr PP (mg/dl) & -0.010 & 0.007 & -0.163 & -1.489 & 0.142 \\
\hline HbA1c \% & 0.410 & 0.276 & 0.212 & 1.482 & 0.144 \\
\hline ACR (mg/g) & 0.006 & 0.001 & 0.537 & 5.865 & $<0.001^{*}$ \\
\hline \multicolumn{6}{|c|}{ Dependent Variable: PODOCALYXIN (ng/ml) } \\
\hline
\end{tabular}

\section{DISCUSSION}

The present study shows that the urinary podocalyxin (PCX) was significantly higher in diabetic patients with macroalbuminuria followed by microalbuminuric group then normoalbuminuric group compared to control group. These results agreed with the study of Hara et al. ${ }^{(7)}$ who found that the urinary podocalyxin was higher in macroalbuminuria than microalbuminuric and normoalbuminuria in study conducted on 71 patients with type two DM . Also these results came in line with the study done by Mohamed et al. (8), which studied the level of podocalyxin in 116 patients with type two DM and they were divided into three groups, group A, with normoalbuminuria, group B with microalbuminuria and group $\mathrm{C}$ with macroalbuminuria and found that type 2 diabetic patients had significant higher levels of urinary podocalyxin in macroalbuminuric group than microalbuminuric group than normoalbuminuric group.

Also these results are in consistent with Ye $\boldsymbol{e t}$ al. (3) who studied 96 subjects, 68 patients with diabetic nephropathy and 28 healthy controls; the patients group were classified into three groups: normoalbuminuria, microalbuminuria and macroalbuminuria. They found that urinary PCX increased in diabetic nephropathy patients with normoalbuminuria compared with the healthy controls, suggesting that podocytes injury occurred before the appearance of microalbuminuria in patients with diabetes and increased significantly in microalbuminuria and macroalbuminuria.

But these results are in disagreement with the study done by Nakamura et al. ${ }^{(9)}$ who studied 50 diabetic patients ( 10 with normoalbuminuria, 15 with microalbuminuria, 15 with macroalbuminuria and 10 with chronic renal failure) and 10 healthy controls. Urinary podocytes were examined by immunofluorescence using monoclonal antibodies against podocalyxin and found that urinary PCX was absent in diabetic patients type 2 with normoalbuminuria but detected in $53 \%$ microalbuminuria and in $80 \%$ macroalbuminuria patients. To explain this conflict, in the current study the delectation of urinary PCX by ELISA method and this method significantly differs from immunofluorescence.

In the present study, we found that there was a highly significant difference regarding glycemic profile (FBS, 2hr PP and HbAlc) in comparing diabetic patients groups: being highest in patients with macroalbuminuria followed by patients with 
microalbuminuria then patients with normoalbuminuric. These results agreed with the study of Mohamed et al. ${ }^{(8)}$ who said that the diabetic patients with marcoalbuminuria were presented with significantly higher $\mathrm{HbA1c}$ in comparing with (normo- and microalbuminuria).

In the current study, there was a statistical highly significant positive correlation between urinary podocalyxin and $\mathrm{HbA1C}$, these result is in consistent with the study conducted by Hara et al. ${ }^{(7)}$ who found that there was a positive correlation between urinary PCX and HbA1C that might indicate that the hyperglycemic state causes worsening of the glomerular capillary-barrier damage.

In the present study, we found that there was a significant correlation between urinary podocalyxin and urinary albumin creatinine ratio (ACR). These results are in consistent with the study of Shoji $\boldsymbol{e t}$ al. (10) who said that urinary PCX is correlated with urinary albumin creatinine ratio.

In the present study, we found that there was a statistical highly significant positive correlation between urinary podocalyxin and serum creatinine, this result disagreed with the studies done by Mohamed et al. ${ }^{(8)}$ who found that there was no correlation between urinary PCX and serum creatinine.

In our study showed a statistical significant positive correlation between urinary podocalyxin and age. This agreed with the study done by Shoji et al. ${ }^{(10)}$ who said that urinary PCX is significantly related to age. Also there was a statistical significant positive correlation between urinary podocalyxin and systolic blood pressure in DM patients with macroalbuminuria, but these results didn't agree with the study done by Hara et al. ${ }^{(7)}$ who said that there was no correlation between urinary PCX and systolic blood pressure.

In our study the multi variant regression analysis between PCX and other measured parameters showed that the albumin creatinine ratio (ACR) is the most independent factors affecting the level of urinary PCX. These matched with the study of Shoji et al. ${ }^{(10)}$ and Mohamed et al. (8) in which the multiple regression analysis showed that ACR was independent related factor influencing urinary PCX level.

\section{CONCLUSION}

In summary, we found that there was a positive significant correlation between urinary PCX and
HBA1C and ACR. So good control of plasma glucose level and life style modification can delay occurrence of diabetic nephropathy and improve prognosis, also urinary podocalyxin seems to be beneficial as an early marker for early stages of diabetic nephropathy in type 2 DM patients.

\section{Conflict of interest:}

The authors declare no conflict of interests. There was no financial funding from any institution. We declare receiving no funds or grants for this study.

\section{REFERENCES}

1. Kharroubi A, Darwish H (2015): Diabetes Mellitus: The Epidemic of the Century. World Journal of Diabetes, 6 (6): 850-67.

2. Tuttle K, Bakris G, Bilous R et al. (2014): Diabetic Kidney Disease: A Report from an ADA Consensus Conference. Am J Kidney Dis., 64(4):510-33.

3. Ye H, Bai X, Gao H et al. (2014): Urinary podocalyxin positive-element occurs in the early stage of diabetic nephropathy and is correlated with a clinical diagnosis of diabetic nephropathy. Journal of Diabetes and Its Complications, 28 (1): 96-100.

4. Casey G, Phillippa J, Liu X et al. (2006): Podocalyxin Variants and Risk of Prostate Cancer and Tumor Aggressiveness. Human Molecular Genetics, 15 (5): $735-$ 41.

5. Odegaard J, Chawla A (2012): Connecting Type 1 and Type 2 Diabetes through Innate Immunity. Cold Spring Harb Perspect Med., 2(3): a007724.

6. Doyonnas R, Kershaw D, Duhme C et al. (2001): Anuria, Omphalocele, and Perinatal Lethality in Mice Lacking the CD34-Related Protein Podocalyxin. The Journal of Experimental Medicine, 194 (1): 13-27.

7. Hara M, Yamagata K, Tomino Y et al. (2012): Urinary Podocalyxin Is an Early Marker for Podocyte Injury in Patients with Diabetes: Establishment of a Highly Sensitive ELISA to Detect Urinary Podocalyxin. Diabetologia, 55 (11): 2913-19.

8. Mohamed A, Heibah H, Ibrahim $\mathrm{H}$ et al. (2016): Urinary Podocalyxin; a Potential New Marker for Early Diabetic Nephropathy in Type 2 Diabetes Mellitus. Indian Journal of Applied Research, 4 (1): 246-50.

9. Nakamura T, Ushiyama C, Suzuki S et al. (2000): Urinary Excretion of Podocytes in Patients with Diabetic Nephropathy. Nephrology Dialysis Transplantation, 15 (9): 1379-83.

10. Shoji M, Kobayashi K, Takemoto M et al. (2016): Urinary Podocalyxin Levels Were Associated with Urinary Albumin Levels among Patients with Diabetes. Biomarkers, 21 (2): 164-67. 